\title{
GaN Single Crystal Habits and Their Relation to GaN Growth Under High Pressure of Nitrogen
}

\author{
Jolanta Prywer $^{1}$ and S. Krukowski ${ }^{2}$ \\ ${ }^{1}$ Institute of Physics, Technical University of ód, \\ ${ }^{2}$ High Pressure Research Center,
}

(Received Friday, July 17, 1998; accepted Wednesday, October 28, 1998)

In the growth of $\mathrm{GaN}$ from nitrogen dissolved in $\mathrm{Ga}$ under high $\mathrm{N}_{2}$ pressure, two main habits are observed: plate-like and needle-like. The plate-like crystals can be divided into those having (0001), $(000 \overline{1})$ and $\{10 \overline{1} 0\}$ faces and those with the additional $\{10 \overline{1} 1\}$ and $\{10 \overline{1} 2\}$ faces. The needle-like crystals belong to three classes: with or without (0001) faces and a third with unusual, star-like needles. The plate-like and needle-like habits and transformation between these habits are discussed in greater detail. It is shown that it is possible to evaluate the relative growth rates corresponding to such transitions.

\section{Introduction}

Growth of high quality single crystals is of primary importance for the development of homoepitaxial techniques for optoelectronic applications. The high pressure solution method is at present the most reliable way to obtain GaN crystals of size up to $15 \mathrm{~mm}$.

In the growth of $\mathrm{GaN}$ from nitrogen dissolved in $\mathrm{Ga}$ under high pressure of nitrogen two main growth habits are observed: plate-like and needle-like [1] [2] [3] [4]. There are a few kinds of plate-like habits. They differ from each other by the pyramidal faces. Among needlelike habits there are also a few kinds which differ from each other by the pyramidal faces and the tips of the needles which may be sharp or blunt. There are also observed habits which are something between the plate and needle. Looking at such transient habits, the question arises whether a transformation from plate-like to needle-like habit is possible.

The aim of this study is to analyze the growth process of both types of GaN single crystals. The attempt to answer the above question is the main subject of this paper.

The computer program SHAPE v. 4.1.1. [5] was chosen as a tool for the graphical presentation of morphologies and sections of GaN crystals (crystal class $6 m m, a=3.1892 \AA, c=5.1850 \AA$ ) and for presentation of the analytically predicted transitions between different habits.

\section{Method of Crystal Growth}

The observed GaN single crystals were grown from a metallic solution of nitrogen in liquid $\mathrm{Ga}$ under high pressure of nitrogen (close to $15 \mathrm{kbar}$ ) and high temperature (close to $1500{ }^{\circ} \mathrm{C}$ ) [2] [4]. In this method GaN single crystals are grown on the sides of the crucible in the cold end zone. The supersaturation is determined from the temperature difference leading to a difference in nitrogen solubility [2] [4].

\section{The Observed GaN Single Crystal Habits}

Generally, GaN single crystal habits we observe may be divided into two kinds. The first one is the plate-like habit (Figure 1a). The second kind is the needle-like habit (Figure 1b). Sometimes, unusual star-like needles were observed.

Generally, it is possible to divide the observed platelike habits into four types which are shown schematically in Figure 2. They differ from each other by the pyramidal faces. We observed platelet habits without any pyramidal faces - Figure 2a. We also observed platelet habits with $\{10 \overline{1} 1\}$ pyramidal faces (Figure $2 \mathrm{~b}$ ) or $\{10 \overline{1} 2\}$ pyramidal faces (Figure 2c). Sometimes these pyramidal faces were observed simultaneously (Figure 2d). The plate-like crystals are characterized by hexagonal shape with relatively small thickness. The morphologies of the two sides of these plate-like crystals are different. One side is almost atomically flat 
whereas the other is full of multiatomic steps with some hill and valley structures.

Generally, we observed three types of needle-like GaN single crystal habits: needle-like habits with $\{10 \overline{1} 1\}$ pyramidal faces (Figure 3a), needle-like habits with $\{10 \overline{1} 2\}$ pyramidal faces (Figure $4 a$ ), or needle-like habits with both these faces observed simultaneously (Figure 4b). The tips of all three types of needle-like habits may be sharp or blunt (cut by the (0001) face or not). We have never observed the needle-like habits without pyramidal faces. In all cases they have (1011) faces present on the high growth rate side. The needlelike crystals are obtained at higher supersaturation. They are characterized by elongated shape.

Generally, plate-like crystals are characterized by much more stable growth. The morphological instabilities of plate-like crystals are mostly related to the edge instability. On the other hand the needle-like crystals frequently show twins or more pronounced instabilities, such as star-shapes. This is related to the characterized growth regimes: it is known that plate-like crystals are observed for lower supersaturations and needle-like for higher supersaturations.

As it is seen in Figure 1b some of the crystals are in the shape of plate, some of them are in the shape of needle and the shape of some of them is something between plate-like and needle-like. That is why it is supposed that it may happen that at first the habits are plate-like. During the growth process the crystals become bigger and some of them remain of the platelet shape but some of them change shape from plate-like to needle-like.

\section{Theoretical Analysis of the Observed GaN Single Crystal Habits}

\subsection{Method of analysis}

In order to investigate how the plate-like or needle-like $\mathrm{GaN}$ single crystals grow, it is necessary to take a closer look at the longitudinal sections along each of the habits (cf. Figure 2). All sections of crystals shown in this paper present the growth bands corresponding to subsequently growing layers, often observed in real crystals. Each layer corresponds to the position of a given face at equal time intervals. It means that the growth bands allow the investigation of the growth history of crystals [6]. Therefore, the sections illustrate growth histories of exemplary GaN single crystals. If we assume that the layer of a given face grows with constant rate, the distances between the growth bands are proportional to growth rates of individual faces. The same layer, but of some other face, grows with constant rate too, but these two constant rates do not have to be the same. It means that the distance between growth bands is not the same for different faces.
In all cases considered in this paper the growth begins from a two-dimensional seed. The (0001) face adheres to a basis and its growth rate is equal to zero. The two-dimensional seed grows and plate-like or needle-like three-dimensional crystals appear. It would be very interesting to know how it happens that the crystal habits that appear are so different.

In order to explain these different crystal habits let us analyze the critical growth rate $R_{A}{ }^{c r i t}$, defined as the normal growth rate of face $A$ at which the edge $l_{A / C}$ created by faces $A$ and $C$ preserves its size [7] [8]. It was derived in [9] that the critical growth rate $R_{A}{ }^{\text {crit }}$ may be expressed by the formula:

$$
R_{A}^{c r i t}=\frac{\sin \beta\left(R_{B} \sin \gamma \sin \psi_{2}+R_{D} \sin \alpha \sin \psi_{1}\right)-R_{C} \sin \alpha \sin \gamma \sin \left(\psi_{1}+\psi_{2}\right)}{\sin \beta\left(\cos \alpha \sin \gamma \sin \psi_{2}+\cos \gamma \sin \alpha \sin \psi_{1}\right)-\cos \beta \sin \gamma \sin \alpha \sin \left(\psi_{1}+\psi_{2}\right)} \text { (1) }
$$

where: $R_{B}, R_{C}$ and $R_{D}$ are the normal growth rates of individual faces $B, C$ and $D$ respectively. These faces $B$, $C$ and $D$ are surrounding faces of the edge $l_{A / C}$. Their growth rates, $R_{B}, R_{C}$ and $R_{D}$, are assumed to be constant not during the whole growth process, but only during the growth of a single layer of crystal. A layer of crystal may be defined as the distance between one growth band and another as described above. The angles $\alpha, \beta, \gamma$ are the angles between vectors normal to the pairs of faces: $A / B, A / C, A / D$, respectively. The angles $\psi_{1}, \psi_{2}$ are the complements of angles between appropriate edges of face $A$ [9]. If the face $A$ grows with normal growth rate $R_{A}$ greater than $R_{A}$ crit, then the size of $l_{A / C}$ decreases. In the case where the growth rate $R_{A}$ is smaller than $R_{A}{ }^{c-}$ ${ }^{r i t}$ the edge size increases. For some faces, (for example all faces without parallel edges or pentagonal faces) the critical growth rate for a given edge is simultaneously the critical growth rate for the whole face [10]. This means that for such cases not only a given edge but also the whole face may change or preserve its size.

The above formula is applied in this paper to $\mathrm{GaN}$ single crystals. It yields critical growth rates for different edges and faces which correspond to decreases in the size of some faces and to increases in the size of other faces. The values obtained for these critical growth rates make it possible to analyze the changes in habits of growing GaN crystals.

\subsection{Transformation from plate-like into nee- dle-like GaN single crystal habits}

In order to investigate how these plate-like or needlelike crystals grow, let us take a closer look at one chosen habit, for example the one shown in Figure 3a and Figure $3 \mathrm{~b}$. The section (Figure $3 \mathrm{~b}$ ) shows the growth bands which illustrate an exemplary growth history and the transition from plate-like to needle-like $\mathrm{GaN}$ single crystal habit. The growth begins from a two-dimen- 
sional seed. The two-dimensional seed grows and a plate-like three-dimensional crystal appears. Up to the $m_{3}$ growth band (Figure $2 \mathrm{~b}$ ) the relative growth rates $R_{\{10 \overline{1} 0\}} / R_{\{10 \overline{1} 1\}}$ and $R_{(0001)} / R_{\{10 \overline{1} 1\}}$ are assumed to be constant and equal to 1.07 and 0.13 , respectively. Beginning from the $m_{3}$ growth band these relative growth rates change. Up to $m_{6}$ growth band the relative growth rate $R_{\{10 \overline{1} 0\}} / R_{\{10 \overline{1} 1\}}$ remains the same but the ratio $R_{(0001)} / R_{\{10 \overline{1} 1\}}$ changes its value to 0.20 . This change is big enough to start the appearance of the $\{10 \overline{1} 1\}$ set of faces $-m_{4}$ growth band - Figure $3 \mathrm{~b}$. From these relative growth rates it is seen that the growth rates of the new $\{10 \overline{1} 1\}$ faces are a little smaller than the rate of the neighboring $\{10 \overline{1} 0\}$ face and is much higher than the growth rate of another neighboring face (0001). The next assumed changes in these relative growth rates (changes in $R_{\{10 \overline{1} 0\}} / R_{\{10 \overline{1} 1\}}$ from 1.02 at the $m_{7}$ growth band to 0.82 at the $m_{15}$ growth band and in $R_{(0001)}$ $R_{\{10 \overline{1} 1\}}$ from 0.51 at the $m_{7}$ growth band to 1.51 at the $m_{15}$ growth band) cause the increase in size of all faces. It is seen that up to the $m_{15}$ growth band the crystal is in a plate-like shape.

It is possible to apply Equation (1) to GaN single crystals and evaluate the relative growth rate $R_{(0001)}^{\text {crit }}$. The (0001) face is surrounded by $\{10 \overline{1} 1\}$ faces. If there is no anisotropy of growth rates of the faces in the $\{10 \overline{1} 1\}$ set surrounding the (0001) face, when this face disappears it becomes a corner. This means that the ratio $R_{0001)}^{\text {crit }}$ corresponds to the size changes of the whole (0001) face, not only to one of its edges. In the case of the (0001) face $R_{B}=R_{C}=R_{D}=R_{\{10 \overline{1} 1\}}$. When we divide the above Equation (1) by $R_{\{10 \overline{1} 1\}}$, it is possible to evaluate the critical relative growth rate, $R_{(0001)}^{c r i t} / R_{\{10} \overline{1}_{1\}}$. For the (0001) face the angles $\alpha=\beta=\gamma=(0001) /$ $\{10 \overline{1} 1\}=61.96^{\circ}$ and $\psi_{1}=\psi_{2}=60.00^{\circ}$. Substituting these values into Equation (1) we have $R_{(0001)}^{c \text { rit }} / R_{\{10 \overline{1} 1\}}$ equal to 2.13. This relative growth rate depends only on the geometry of the crystal represented by the appropriate angles. The value of this critical relative growth rate means that for relative growth rate $R_{(0001)}$ $R_{\{10 \overline{1} 1\}}=R_{(0001)}^{\text {crit }} / R_{\{10 \overline{1} 1\}}=2.13$, the size of the $(0001)$ face remains the same during further growth. Up to the $m_{15}$ growth band the ratio $R_{(0001)} / R_{\{10 \overline{1} 1\}}$ is smaller than the evaluated value 2.13 and the sector of the (0001) face is wide. However, beginning from the $m_{16}$ growth band, the (0001) face grows very fast $\left(R_{(0001)}\right.$
$\left.R_{\{10 \overline{1} 1\}}=2.86\right)$ while the $\{10 \overline{1} 0\}$ face grows more and more slowly $\left(R_{\{10 \overline{1} 0\}} / R_{\{10 \overline{1} 1\}}=0.61\right)$ and the crystal starts to transform to the needle-like habit. As a result of the further increase of growth rate of the (0001) face this face disappears in the habit at the $m_{27}$ growth band and it is absent in the $m_{28}$ growth band. Finally, the face is present in the habit because of the decrease of relative growth rate $R_{(0001)} / R_{\{10 \overline{1} 1\}}$ to $1.22\left(R_{\{10 \overline{1} 0\}} / R_{\{10 \overline{1} 1\}}=\right.$ 0.31). The final habit is needle-like.

Similarly, as it was done for the (0001) face, it is also possible to evaluate $R_{\{10 \overline{1} 0\}}^{c \text { rit }} / R_{\{10 \overline{1} 1\}}$. In this case face $B$ is the face belonging to the $\{10 \overline{1} 1\}$ set of faces, so $R_{B}=R_{\{10 \overline{1} 1\}}$. Face $C$ is the face belonging to the $\{10 \overline{1} 1\}$ set of faces, so $R_{C}=R_{\{10 \overline{1} 0\}}$. Face $D$ corresponds to the $(000 \overline{1})$ face, whose growth rate was assumed to be equal to zero. The angle $\alpha$ is the angle between vectors normal to two adjacent faces, the first one from the $\{10 \overline{1} 1\}$ set and the second from the $\{10 \overline{1} 0\}$ set and it is equal to $28.04^{\circ}$. The angle $\beta$ is the angle between vectors normal to two neighboring faces belonging to the set $\{10 \overline{1} 0\}$ and it is equal to $60.00^{\circ}$. The angle $\gamma$ is the angle between a vector normal to a face from the $\{10 \overline{1} 0\}$ set and one normal to the $(000 \overline{1})$ face and it is equal to $90.00^{\circ}$. As the faces $\{10 \overline{1} 0\}$ are of rectangular shape the angles $\psi_{1}, \psi_{2}$ are the same and equal to $90.00^{\circ}$. Because the sum of $\psi_{1}, \psi_{2}$ is equal to $180^{\circ}$, $R_{\{10 \overline{1} 0\}}^{c \text { rit }} / R_{\{10 \overline{1} 1\}}$ does not depend on the $R_{C}$ growth rate (see Equation (1)). In such a case the ratio $R_{\{10 \overline{1} 0\}}^{c+i t} / R_{\{10 \overline{1} 1\}}$ depends on the geometry of crystal and is equal to 1.13 . The relative growth rate $R_{\{10 \overline{1} 0\}}^{\text {crit }} / R_{\{10 \overline{1} 1\}}$ is smaller than the evaluated value 1.13 throughout the considered exemplary GaN single crystal growth process. As a result, the $\{10 \overline{1} 0\}$ face becomes bigger and bigger and in the final habit this set of faces is very well developed.

Let us take a closer look at the habit at the $m_{9}$ growth band. It is seen that at this stage of growth the crystal is plate-like. The relative growth rates $R_{(0001)} / R_{\{10 \overline{1} 1\}}$ and $R_{\{10 \overline{1} 0\}} / R_{\{10 \overline{1} 1\}}$ for this growth band are assumed to be equal to 0.51 and 1.01 , respectively. If these relative growth rates were constant from this band till the end of growth process, the final shape of crystal would be plate-like, very similar to that seen in Figure 1b or Figure 2 .

Generally, it is seen that the plate-like GaN single crystals grow when the relative growth rate $R_{(0001)}$ $R_{\{10 \overline{1} 1\}}$ is much smaller than the evaluated critical 
ratio $R_{(0001)}^{\text {crit }} / R_{\{10 \overline{1} 1\}}=2.13$ and also when the relative growth rate $R_{\{10 \overline{1} 0\}} / R_{\{10 \overline{1} 1\}}$ is a little smaller than $R_{\{10 \overline{1} 0\}}^{c+i t} / R_{\{10 \overline{1} 1\}}=1.13$. Such values of relative growth rates guarantee a continuous increase in the (0001) face size and a moderate increase in the $\{10 \overline{1} 0\}$ face size.

For some crystals, pyramids are formed with $\{10 \overline{1} 1\}$ faces and for others, with $\{10 \overline{1} 2\}$ faces (Figure 4). In the latter case it is necessary to consider $R_{\{0001\}}^{\text {crit }} / R_{\{10 \overline{1} 2\}}$ and $R_{\{10 \overline{1} 0\}}^{\text {crit }} / R_{\{10 \overline{1} 2\}}$. The first value $R_{\{0001\}}^{\text {crit }} / R_{\{10 \overline{1} 2\}}$ is equal to $1.37\left(R_{B}=R_{C}=R_{D}=R_{\{10 \overline{1} 2\}}\right.$, $\alpha=\beta=\gamma=(0001) /\{10 \overline{1} 2\}=43.19^{\circ}, \psi_{1}=\psi_{2}=$ $\left.60.00^{\circ}\right)$. The relative growth rate $R_{\{10 \overline{1} 0\}}^{\text {crit }} / R_{\{10 \overline{1} 2\}}$ is equal to 1.46 . To determine this value it is necessary to know that the faces $B$ and $C$ are the faces belonging to the $\{10 \overline{1} 2\}$ set and the $\{10 \overline{1} 0\}$ set, respectively. Therefore, $R_{B}=R_{\{10 \overline{1} 2\}}$ and $R_{C}=R_{\{10 \overline{1} 0\}}$. Face $D$ corresponds to $(000 \overline{1})$, thus, $R_{D}=R_{(000 \overline{1})}=0$. The angle $\alpha$ is the angle between normals to two adjacent faces, the first one from the $\{10 \overline{1} 0\}$ set and the second from the $\{10 \overline{1} 2\}$ set and is equal to $46.81^{\circ}$. The angle $\beta$ is the angle between normals to two neighboring $\{10 \overline{1} 0\}$ faces and is equal to $60.00^{\circ}$. The angle $\gamma$ is the angle between a vectors normal to a $\{10 \overline{1} 0\}$ face and a $(000 \overline{1})$ face and is equal to $90.00^{\circ}$. The angles $\psi_{1}, \psi_{2}$ are both equal to $90.00^{\circ}$. These values, $\quad R_{\{0001\}}^{\text {crit }} / R_{\{10 \overline{1} 2\}}=1.37$ and $R_{\{10 \overline{1} 0\}}^{\text {crit }} / R_{\{10 \overline{1} 2\}}=1.46$ indicate that the plate-like GaN single crystals with pyramidal faces $\{10 \overline{1} 2\}$ grow when the relative growth rate $R_{(0001)} / R_{\{10 \overline{12}\}}$ is much smaller than 1.37 and when $R_{\{10 \overline{1} 0\}} / R_{\{10 \overline{1} 2\}}$ a little smaller than 1.46. Such values of relative growth rates guarantee successive increase in the (0001) face size and a moderate increase of the $\{10 \overline{1} 0\}$ face size.

In the case considered above it is assumed that all faces belonging to a given set $\{10 \overline{1} 0\},\{10 \overline{1} 1\}$ or $\{10 \overline{1} 2\}$ grow with the same rates. This assumption was made for simplicity. But in real crystals the growth rates of faces from the same set may differ slightly. In such cases the hexagonal symmetry is disordered as it may be seen in Figure 1a.

Sometimes, star-like GaN crystal habits were observed - Figure 5. In this figure well developed $\{10 \overline{1} 0\}$ faces which forming a star created by raised edges of $\{10 \overline{1} 1\}$ faces may be seen. If the faces were absent in the habit, it would be possible to suppose that the star-like crystal is in fact a triplet crystal. But when the star-like crystals were observed, they always were surrounded by the $\{10 \overline{1} 0\}$ faces. For that reason it is supposed that habits like this are the result of unstable growth [11]. The most likely reason for growth of such crystals is that the higher supersaturation at the corners and edges of the crystal leads to an increase in growth rate with increasing distance from the center of the face. At first such progressive increase in supersaturation leads to more rapid growth of edge and corner regions and as a result raised edges develop. Simultaneously, the center of the faces tend to develop terraced depressions. The next stage is the hopper crystals and, in the extreme case of growth at the edges of crystal, the final shape will be dendritic. In the considered case of GaN single crystals, the dendritic shape is sometimes seen in the direction of rapid growth. The slowly growing faces are very well developed and without depressions.

On the basis of the above analysis and the exemplary longitudinal sections of GaN single crystal habits, the conclusion may be drawn that the transition from the plate-like to needle-like habit is possible and it can occur in a stable way. The transformation from the plate-like into the needle-like habit in the case of the $\{10 \overline{1} 1\}$ pyramidal faces is caused by the increase in the relative growth rate $R_{(0001)} / R_{\{10 \overline{1} 1\}}$ above 2.13 and a simultaneous decrease in the relative growth rate $R_{\{10 \overline{1} 0\}} / R_{\{10 \overline{1} 1\}}$ below 1.13 . When the pyramid is created by the set of $\{10 \overline{1} 2\}$ faces, the transformation from plate-like to needle-like habit is caused by the increase in the relative growth rate $R_{(0001)} / R_{\{10 \overline{1} 2\}}$ above 1.37 and simultaneous decrease in relative growth rate $R_{\{10 \overline{10}\}^{\prime}} / R_{\{10 \overline{1} 2\}}$ below 1.46 . The observed star-like needles are supposed to be the result of unstable growth. The higher supersaturation at the corners and edges of crystal leads to the more rapid growth at these regions and, as a result, the final shape is star-like.

\section{Conclusions}

Our considerations allow us to draw the following conclusions:

1. GaN growth habits depend on the relative growth rates; the transition from plate-like to needle-like habits may occur without loss of morphological stability.

2. The transformation from plate-like to needle-like habit, in the case when the pyramid is created by the set of the $\{10 \overline{1} 1\}$ faces, is caused by the increase in the relative growth rate $R_{(0001)} / R_{\{10 \overline{1} 1\}}$ above the evaluated value 2.13 and simultaneous decrease in the relative growth rate $R_{\{10 \overline{10} 0} / R_{\{10 \overline{1} 1\}}$ below the value 1.13 .

3. When the pyramid is created by the set of $\{10 \overline{1} 2\}$ faces, the transformation from plate-like to needle-like habit is caused by the increase in the relative growth rate $R_{(0001)} / R_{\{10 \overline{1} 2\}}$ above the evaluated value 1.37 and the 
simultaneous decrease in relative growth rate $R_{\{10 \overline{10}\}}$ $R_{\{10 \overline{1} 2\}}$ below the value 1.46 .

4. GaN star-like crystals result from unstable morphologically growth rate.

5. The relative change of the growth rates occurs via the kinetic mechanism and depends on the orientation of the growing face. The change of the mechanism can lead to growth of three-dimensional GaN crystals.

\section{REFERENCES}

[1] D. Elwell, R. S. Feigelson, M. M. Simkins, W. A. Tiller, J. Cryst. Growth 66, 45 (1984).

[2] I. Grzegory, S. Krukowski, Phys. Scr. T39 , 242-249 (1991).

[3] H. Yamane, M. Shimada, T. Sekiguchi, F. J. DiSalvo, J. Cryst. Growth 186, 8 (1998).

[4] I Grzegory, J Jun, S Krukowski, et al., Physica B 185 , 99-102 (1993).

[5] E. Dowty, Am. Miner. 65, 627 (1980).

[6] P. Görnert and F. Voigt in: Current Topics in Materials Science, vol. 11, ed. E. Kaldis North-Holland, Amsterdam, 1984, ch. 1

[7] M. Szurgot, J. Prywer, Cryst. Res. and Tech. 26, 147 (1991).

[8] M. Szurgot, Cryst. Res. and Tech. 27, 919 (1992).

[9] J Prywer, J. Cryst. Growth 158, 568 (1996).

[10] J. Prywer, J. Cryst. Growth 165 , 335 (1996).

[11] D. Elwell, H. J. Scheel in: Crystal Growth from HighTemperature Solutions, Academic Press, New York, 1975

\section{FIGURES}

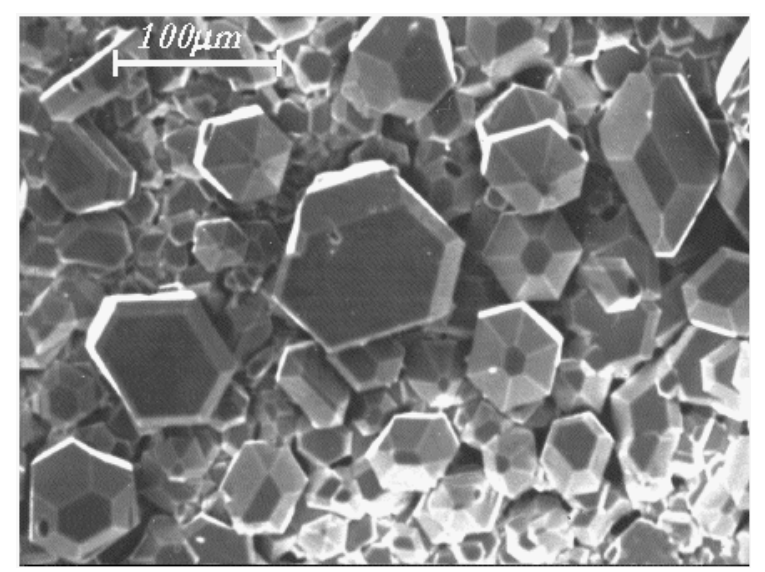

Figure 1a. The exemplary GaN needle-like and plate-like single crystal habits.

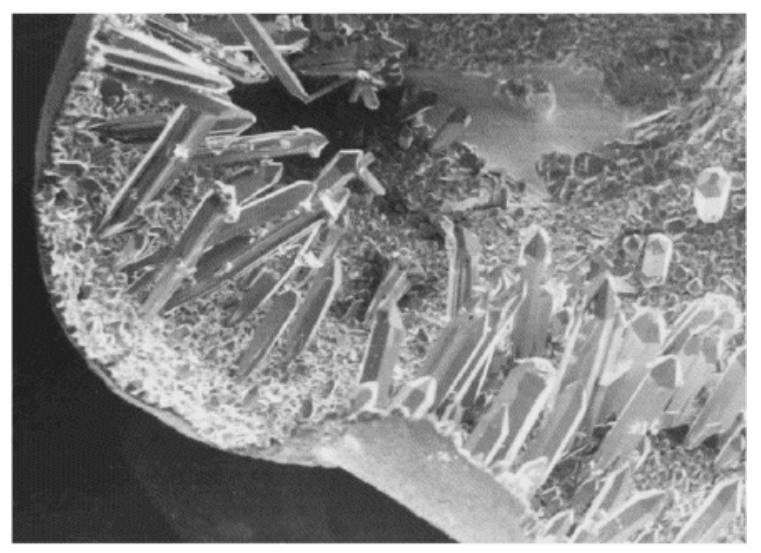

Figure $1 b$. The exemplary GaN needle-like single crystal habits $[2]$.

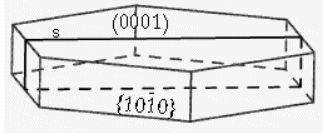

a)

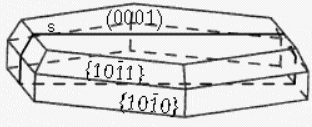

b)

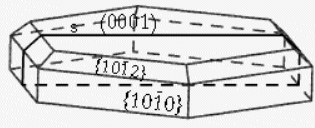

c)
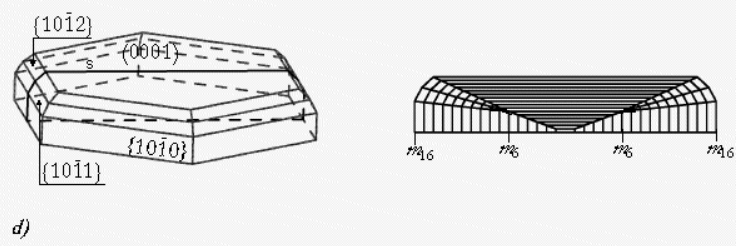

Figure 2. The observed GaN plate-like single crystal habits and their longitudinal sections; habits: $a$ ) without pyramidal faces, $b)$ with pyramidal faces $\{10 \overline{1} 1\}, c)$ with pyramidal faces $\{10 \overline{1} 2\}, d)$ with both sets of pyramidal faces. The letter "s" indicates the line of longitudinal section; $m_{6}, m_{16}$ denote growth bands. 


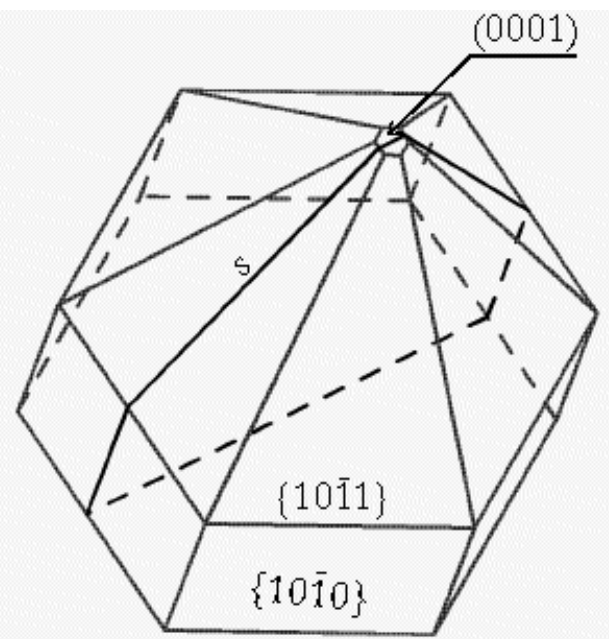

Figure 3a. The observed GaN needle-like single crystal habits with pyramidal faces $\{10 \overline{1} 1\}$. The letter "s" indicates the line of longitudinal section shown in Figure $3 b$.

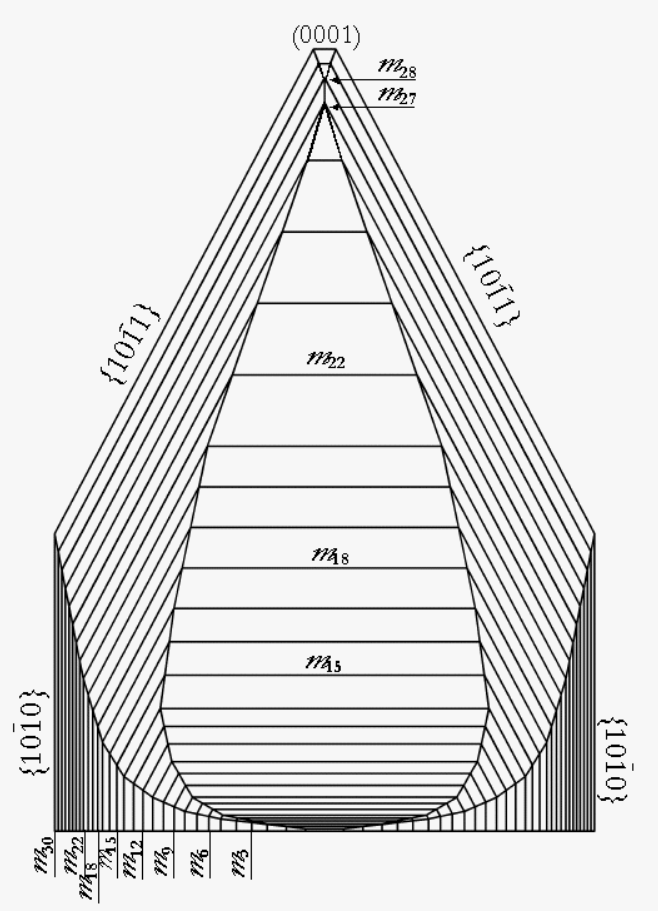

Figure $3 \mathrm{~b}$. The longitudinal section of $\mathrm{GaN}$ single crystal habit shown in Figure $3 \mathrm{a}$, with pyramidal faces $\{10 \overline{1} 1\} . m_{3}, m_{30}$ denote growth bands.
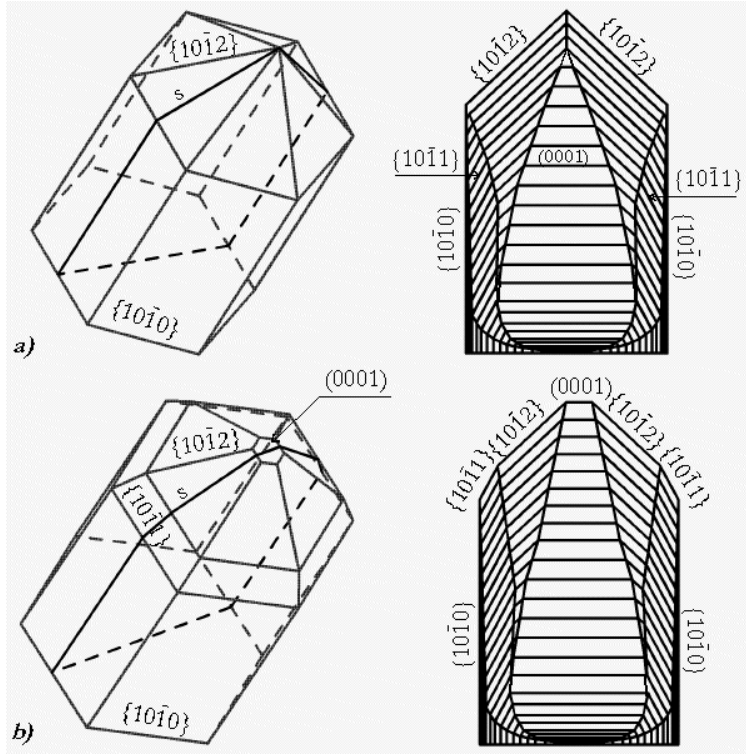

Figure 4. Two kinds of the observed GaN needle-like single crystal habits and their longitudinal sections; habits: $a$ ) with pyramidal faces $\{10 \overline{1} 2\}, b$ ) with both sets of pyramidal faces $\{10 \overline{1} 1\}$ and $\{10 \overline{1} 2\}$. The letter "s" indicates the line of longitudinal section.

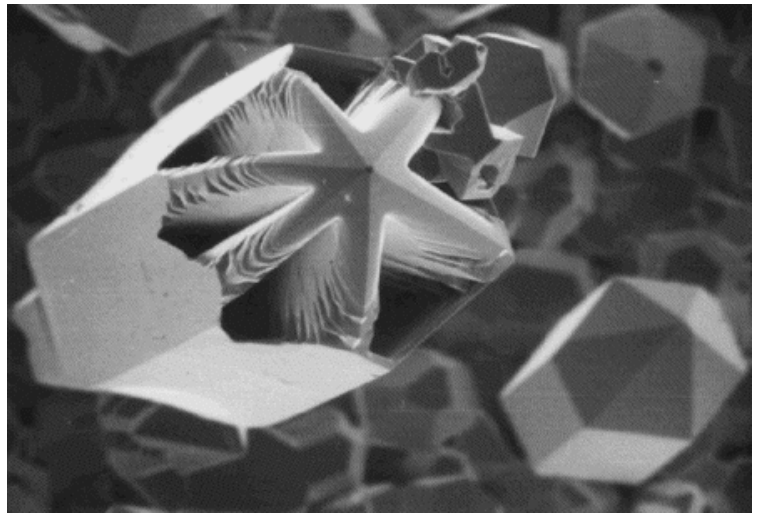

Figure 5. The exemplary star-like GaN single crystal habit [2]. 